УДК 517.5

\title{
В.Л. Великин
}

Днепропетровский науиональный университет имени Олеся Гончара

\section{О ВЗАИМНОМ УКЛОНЕНИИ НЕКОТОРЫХ ПОДПРОСТРАНСТВ ИНТЕРПОЛЯЩИОННЫХ ЭРМИТОВЫХ СПЛАЙНОВ ПО РАЗНЫМ СЕТКАМ УЗЛОВ}

Отримано точні значення інтерполяційних рөзхилів деяких підпросторів ермітових сплайнів за різними сітками вузлів на множинах неперервно диференхійовних функиій.

Ключові слова: розхил, підпростір, сплайни.

Получены точные значения интерполяционных растворов некоторых подпространств эрмитовых сплайнов по разным сеткам узлов на множествах непрерывно дифферекцируемых функций.

Ключевые слова: раствор, подпространство, сплайны.

We obtain exact values on the interpolatory openings of certain subspaces of Hermitian splines for different knots over sets of continuosly differentiable functions.

Key words: openings, subsets, splines.

Пусть $\mathrm{C}^{q}, \mathrm{q}=0,1,2, \ldots$, - линейное нормированное пространство функций $f(x)$, имеющих на промежутке $[0,1]$ q непрерывных производных с нормой

$$
\|\mathbf{f}\|^{(q)}=\sum_{i=0}^{q}\left\|f^{(i)}\right\|_{C_{[0,1]}},\|f\|^{(0)}=\|f\| \text {. }
$$

Пусть еще $\Delta_{v}=\left\{x_{i}^{v}\right\}_{i=0}^{v}, \quad 0=x_{0}^{v}<x_{1}^{v}<\ldots<x_{v}^{v}=1, v \geq 1$, - произвольное разбиение промежутка $[0,1]$ узлами $\mathrm{x}_{\mathrm{i}}^{\mathrm{y}}$.

Положим

$$
h_{i}^{v}=x_{i}^{v}-x_{i-1}^{v},
$$$$
\delta^{v}=\max \left\{h_{i}^{v}: i=\overline{1, n}\right\},
$$$$
\delta_{i}^{v}=\left(x_{i-1}, x_{i}\right)
$$
$\bar{\delta}_{i}^{v}=\left[x_{i-1}^{v}, x_{i}^{v}\right]$.

Каждой функции $f \in \mathrm{C}^{q}$ поставим в соответствие интерполяционный эрмитовый сплайн порядка $2 \mathrm{~m}+1, \mathrm{~m}=0,1,2, \ldots$, вида

$$
\begin{gathered}
s_{r, m}\left(f, \Delta_{v} ; x\right)=\sum_{k=0}^{r} f^{(k)}\left(x_{i-1}^{v}\right) H_{k, m}\left(h_{i}^{v} ; x-x_{i-1}^{v}\right)+(-1)^{k} f^{(k)}\left(x_{i}^{v}\right) H_{k, m}\left(h_{i}^{v} ; x_{i}^{v}-x\right), \\
x \in \bar{\delta}_{i}^{v}, \quad i=\overline{1, n}, \quad q, m \geq r, \quad \text { и } \\
H_{k, m}(h ; t)=\frac{(h-t)^{m+1}}{k ! m !} \sum_{s=0}^{m-k} \frac{(m+s) !}{s ! h^{k+s+1}} t^{k+s}
\end{gathered}
$$

Подпространство таких сплайнов при фиксированных $\mathrm{m}, \mathrm{r}$ и $\Delta_{v}$ обозначим через $\mathrm{S}_{\mathrm{r}, \mathrm{m}}\left(\Delta_{v}\right)$. 
В [1-3] исследовалась величина, представляющая собой, так называемый, интерполяционный раствор пары подпространств эрмитовых сплайнов по одному и тому же набору узлов. Здесь мы продолжаем исследование аналогичной величины для подпространств интерполяционных эрмитовых сплайнов уже по различным наборам узлов.

А именно, положим

$$
\Theta_{\mathrm{r}, \mathrm{p}}\left[\mathrm{S}_{\mathrm{r}_{1}, \mathrm{~m}_{1}}\left(\Delta_{v_{1}}\right) ; \mathrm{S}_{\mathrm{r}_{2}, \mathrm{~m}_{2}}\left(\Delta_{v_{2}}\right)\right]=\sup _{\|f\|^{(r)} \leq 1}\left\|S_{\mathrm{r}_{1}, \mathrm{~m}_{1}}\left(\mathrm{f}, \Delta_{v_{1}} ; \mathrm{x}\right)-\mathrm{s}_{\mathrm{r}_{2}, \mathrm{~m}_{2},}\left(\mathrm{f}, \Delta_{v_{2}} ; \mathrm{x}\right)\right\|_{\mathrm{p}},
$$

где $\mathrm{r} \geq 0$, a $\|\cdot\|_{p}$ - это норма пространства $\mathrm{L}_{\mathrm{p}}(0,1), 1 \leq \mathrm{p} \leq \infty$.

В случае $\mathrm{r}_{1}=\mathrm{r}_{2}=0$ левую часть предыдущего равенства будем обозначать через $\Theta_{\mathrm{r}, \mathrm{p}}\left[\left(\mathrm{m}_{1}, v_{1}\right) ;\left(\mathrm{m}_{2}, v_{2}\right)\right]$.

Положим еще

$$
\mathrm{e}_{v_{1}, v_{2}}^{m_{1}, m_{2}}=s_{0, m_{1}}\left(f, \Delta_{v_{1}} ; x\right)-s_{0, m_{2}},\left(f, \Delta_{v_{2}} ; x\right)
$$

В дальнейшем мы здесь используем две леммы из [1], которые сформулируем ниже.

Лемма A. $\lim _{\mathrm{m} \rightarrow \infty} \mathrm{H}_{0, \mathrm{~m}}(\mathrm{~h} ; \mathrm{t})=\left\{\begin{array}{lr}1, & 0 \leq \mathrm{t}<\mathrm{h} / 2, \\ 1 / 2, & \mathrm{t}=\mathrm{h} / 2, \\ 0, & \mathrm{~h} / 2<\mathrm{t} \leq 1 .\end{array}\right.$

Лемма В. Для любых $\mathrm{x}, \mathrm{x}+\mathrm{h} \in[0,1]$, справедливо равенство

$$
\sup \left\{|f(x)-f(x+h)|: \quad\|f\|^{(1)} \leq 1\right\}=2 h(2+h)^{-1}=a(h) .
$$

Теорема 1. Для любых $\mathrm{m}_{\mathrm{i}} \geq 0, v_{\mathrm{i}} \geq 1, \mathrm{i}=1,2, \mathrm{v}_{1} \neq \mathrm{v}_{2}$, имеет место равенство

$$
\Theta_{0, \infty}\left[\left(m_{1}, v_{1}\right) ;\left(m_{2}, v_{2}\right)\right]=2 .
$$

Доказательство. Как уже отмечалось в [1] (см. также [2]),

$$
\begin{gathered}
\mathrm{H}_{\mathrm{k}, \mathrm{m}}^{(\mathrm{j})}(\mathrm{h} ; 0)=\delta_{\mathrm{k}, \mathrm{j}}, \mathrm{H}_{\mathrm{k}, \mathrm{m}}^{(\mathrm{j})}(\mathrm{h} ; \mathrm{h})=0, \mathrm{j}=\overline{0, \mathrm{~m}, \text { и }} \\
\mathrm{H}_{0, \mathrm{~m}}(\mathrm{~h} ; \mathrm{t})+\mathrm{H}_{0, \mathrm{~m}}(\mathrm{~h} ; \mathrm{h}-\mathrm{t}) \equiv 1,
\end{gathered}
$$

а также

$$
\frac{h-t}{h}=H_{0,0}(h ; t)<H_{0, m}(h ; t)<H_{0, m+1}(h ; t)<1, t \in(0, h / 2), m \in N .
$$

Откуда вытекает следующее соотношение для $\mathrm{x} \in \bar{\delta}_{\mathrm{i}}^{\mathrm{v}}, \mathrm{i}=\overline{1, n}$, :

$$
-1 \leq \min \left\{f\left(x_{i-1}^{v}\right), f\left(x_{i}^{v}\right)\right\} \leq s_{0, m}\left(f, \Delta_{v} ; x\right) \leq \max \left\{f\left(x_{i-1}^{v}\right), f\left(x_{i}^{v}\right)\right\} \leq 1 .
$$

Поэтому

$$
\left\|\mathrm{e}_{v_{1}, v_{2}}^{\mathrm{m}_{1}, \mathrm{~m}_{2}}(\mathrm{f} ; \mathrm{x})\right\|_{\infty} \leq 2
$$

Поскольку $\Delta_{v_{1}} \neq \Delta_{v_{2}}$, то один из интервалов этих двух разбиений содержит узлы другого разбиения. Пусть, например, интервал $\delta_{j}^{v_{1}}$ содержит узел $\mathrm{x}_{\mathrm{i}}^{\mathrm{v}_{2}}$. Тогда для функции $\mathrm{f}_{0} \in \mathrm{C}$, представляющей собой ломаную с изломами в точках $\mathrm{x}_{j-1}^{v_{1}}, \mathrm{x}_{i}^{v_{2}}, \mathrm{x}_{j}^{v_{1}}$ и такой, что $\mathrm{f}_{0}(0)=\mathrm{f}_{0}\left(\mathrm{x}_{\mathrm{j}-1}^{v_{1}}\right)=\mathrm{f}_{0}\left(\mathrm{x}_{\mathrm{j}}^{v_{1}}\right)=\mathrm{f}_{0}(1)=-1, \mathrm{f}_{0}\left(\mathrm{x}_{\mathrm{i}}^{v_{2}}\right)=1$, 
имеем

$$
\begin{gathered}
\left\|\mathrm{e}_{v_{1}, v_{2}}^{\mathrm{m}_{1}, \mathrm{~m}_{2}}\left(\mathrm{f}_{0} ; \mathrm{x}\right)\right\|_{\mathrm{C}\left[\bar{\delta}_{j}^{v_{1}}\right]}=\left\|-1-\mathrm{s}_{0, \mathrm{~m}_{2}}\left(\mathrm{f}_{0}, \Delta_{v_{2}} ; \mathrm{x}\right)\right\|_{\mathrm{C}\left[\bar{\delta}_{j}^{-\gamma_{1}}\right]} \geq \\
\geq 1-1-\mathrm{s}_{0, \mathrm{~m}_{2}}\left(\mathrm{f}_{0}, \Delta_{v_{2}} ; \mathrm{x}_{\mathrm{i}}^{v_{2}}\right)|=|-1-\mathrm{f}_{0}\left(\mathrm{x}_{\mathrm{i}}^{v_{2}}\right) \mid=2 .
\end{gathered}
$$

Теорема 1 доказана.

Далее рассмотрим два произвольных разбиения $\Delta_{n}$ и $\Delta_{n+s}, s \geq 1$, таких, что $\Delta_{n} \subset \Delta_{n+s}$. Обозначим через $\omega_{1}=\omega_{1}\left(\Delta_{n}\right)$ - множество индексов ј тех интервалов $\delta_{j}^{n}$, которые не содержат узлов $\Delta_{n+s}$, а через $\omega_{2}=\omega_{2}\left(\Delta_{n}\right)$ множество $\{1,2, \ldots, n\} \backslash \omega_{1}$. Для $\mathrm{j} \in \omega_{2}$ положим

$\bar{x}_{j}^{n+s}=\min \left\{x_{i}^{n+s}: x_{j-1}^{n}<x_{i}^{n+s}<x_{j}^{n}\right\}, \quad \bar{x}_{j}^{n+s}=\max \left\{x_{i}^{n+s}: x_{j-1}^{n}<x_{i}^{n+s}<x_{j}^{n}\right\}$.

Здесь нам понадобится еще функция $\varphi_{\mathrm{m}}$ из [1]

$$
\varphi_{\mathrm{m}}(\mathrm{t})=\mathrm{H}_{0, \mathrm{~m}+1}(1 ; \mathrm{t})-\mathrm{H}_{0, \mathrm{~m}}(1 ; \mathrm{t})=\mathrm{C}_{2 \mathrm{~m}+1}^{\mathrm{m}} \mathrm{t}^{\mathrm{m}+1}(1-\mathrm{t})^{\mathrm{m}+1}(1-2 \mathrm{t}),
$$

для которой

$$
\int_{0}^{1 / 2} \varphi_{m}(\mathrm{t}) \mathrm{dt}=\frac{(2 \mathrm{~m}+1) !}{2^{2 \mathrm{~m}+2} \mathrm{~m} !(\mathrm{m}+2) !}:=\Phi(\mathrm{m}) .
$$

Теорема 2. Для любых $\mathrm{m}_{\mathrm{i}} \geq 0, \mathrm{i}=1,2, u \Delta_{\mathrm{n}} \subset \Delta_{\mathrm{n}+\mathrm{s}}, \mathrm{s} \geq 1$, имеет место равенство

$$
\Theta_{0,1}\left[\left(m_{1}, n\right) ;\left(m_{2}, n+s\right)\right]=\sum_{\mu=1}^{m_{2}-m_{i}} \Phi\left(m_{1}+\mu-1\right) \sum_{j \in \omega_{1}} h_{j}^{n}+\sum_{j \in \Theta_{2}}\left(h_{j}^{n}+\overline{\bar{x}}_{j}^{n+s}-\bar{x}_{j}^{n+s}\right) .
$$

Доказательство. Пусть сначала интервал $\delta_{\mathrm{j}}^{\mathrm{n}}$ не содержит узлов разбиения $\Delta_{n+s}$, т.е. $j \in \omega_{1}$. В этом случае поступим, с учетом соотношений (4) и (1), так же, как и при доказательстве теоремы 4 из [1]. А именно,

$$
\begin{gathered}
\int_{x_{j-1}^{n}}^{x_{j}^{n}}\left|e_{n, n+s}^{m_{1}, m_{2}}(f ; x)\right| d x \leq 2 \int_{0}^{h_{j}^{n}}\left|H_{0, m_{2}}\left(h_{j}^{n} ; t\right)-H_{0, m_{1}}\left(h_{j}^{n} ; t\right)\right| d t= \\
=2 \sum_{\mu=1}^{m_{2}-m_{1} h_{j}^{n}} \int_{0}^{h_{0, m_{i}+\mu}}\left(h_{j}^{n} ; t\right)-H_{0 ; m_{1}+\mu-1}\left(h_{j}^{n} ; t\right) \mid d t=4 h_{j}^{n} \sum_{\mu=1}^{m_{2}-m_{1}} \int_{0}^{1 / 2} \varphi_{m_{1}+\mu-1}(t) d t= \\
=h_{j}^{n} \sum_{\mu=1}^{m_{2}-m_{1}} \Phi\left(m_{1}+\mu-1\right) .
\end{gathered}
$$

Знак равенства в соотношении (6) достигается для непрерывной функции, которая в узлах $\mathrm{x}_{j-1}^{\mathrm{n}}, \mathrm{x}_{j}^{\mathrm{n}}$ принимает значения -1 и 1 соответственно (или 1 и -1). Если же интервал $\delta_{j}^{n}$ содержит узлы разбиения $\Delta_{n+s}$, т.е. $j \in \omega_{2}$, то

$$
\begin{gathered}
\int_{x_{j-1}^{n}}^{x_{j}^{n}} e_{n, n+s}^{m_{1}, m_{2}}(f ; x) \mid d x \leq 2 \int_{x_{j-1}^{n}}^{\bar{x}_{j}^{n+s}} \frac{x-x_{j-1}^{n}}{\bar{x}_{j}^{n+s}-x_{j}^{n}} d x+2 \int_{\bar{x}_{j}^{n+s}}^{\bar{x}_{j}^{n+s}} d x+2 \int_{\overline{\bar{x}}_{j}^{n+s}}^{x_{j}^{n}} \frac{x_{j}^{n}-x}{x_{j}^{n}-\overline{\bar{x}}_{j}^{n+s}} d x= \\
=h_{j}^{n}+\overline{\bar{x}}_{j}^{n+s}-\bar{x}_{j}^{n+s}
\end{gathered}
$$


Причем знак равенства реализует непрерывная функция, которая в точках $\mathrm{x}_{\mathrm{j}-1}^{\mathrm{n}}, \quad \overline{\mathrm{x}}_{\mathrm{j}}^{\mathrm{n}+\mathrm{s}}, \overline{\overline{\mathrm{x}}}_{\mathrm{j}}^{\mathrm{n}+\mathrm{s}}, \mathrm{x}_{\mathrm{j}}^{\mathrm{n}}$ принимает значения $-1,1,1$ и -1 соответственно (или 1 , $-1,-1$ и 1$)$.

Остается отметить, что функция $\mathrm{f} \in \mathrm{C}$, которая реализует знак равенства в (6) на каждом промежутке $\bar{\delta}_{j}^{n}$, для $\mathrm{j} \in \omega_{1}$, и в (7), для $\mathrm{j} \in \omega_{2}$, будет экстремальной на всем промежутке $[0,1]$.

Следствие. Имеет место соотношение

$$
\begin{gathered}
\sum_{\mu=1}^{m_{2}-m_{1}} \Phi\left(m_{1}+\mu-1\right) \sum_{j \in \omega_{1}} h_{j}^{n}+\sum_{j \in \omega_{2}} h_{j}^{n} \leq \Theta_{0,1}\left[\left(m_{1}, n\right) ;\left(m_{2}, n+s\right)\right]< \\
<\sum_{\mu=1}^{m_{2}-m_{1}} \Phi\left(m_{1}+\mu-1\right) \sum_{j \in \omega_{1}} h_{j}^{n}+2 \sum_{j \in \omega_{2}} h_{j}^{n} .
\end{gathered}
$$

В частности, если каждый интервал $\delta_{\mathrm{j}}^{\mathrm{n}}$ содержит узлы разбиения $\Delta_{\mathrm{n}+\mathrm{s}}$, то

$$
1 \leq \Theta_{0,1}\left[\left(m_{1}, n\right) ;\left(m_{2}, n+s\right)\right]<2 .
$$

Причем константа 2 справа не уменьшаема на множестве всех разбиений $\Delta_{n+s}$. А если каждый интервал $\delta_{j}^{n}$ содержит в точности один узел разбиения $\Delta_{2 n}$, то

$$
\lim _{m_{1}, m_{2} \rightarrow \infty} \Theta_{0,1}\left[\left(m_{1}, n\right) ;\left(m_{2}, n+s\right)\right]=1
$$

Теорема 3. Для любых $\mathrm{m}_{\mathrm{i}} \geq 0, \mathrm{i}=1,2, \Delta_{\mathrm{n}} \subset \Delta_{\mathrm{n}+\mathrm{s}} u \mathrm{p} \geq 1$,

$$
\lim _{\mathrm{m}_{1}, \mathrm{~m}_{2} \rightarrow \infty} \Theta_{0, \mathrm{p}}\left[\left(\mathrm{m}_{1}, \mathrm{n}\right) ;\left(\mathrm{m}_{2}, \mathrm{n}+\mathrm{s}\right)\right] \leq\left(\frac{1}{\mathrm{p}+1} \sum_{\mathrm{j} \in \omega_{1}}\left(\mathrm{~h}_{\mathrm{j}}^{\mathrm{n}}\right)^{\mathrm{p}+1}+2^{\mathrm{p}} \sum_{\mathrm{j} \in \omega_{2}} \mathrm{~h}_{\mathrm{j}}^{\mathrm{n}}\right)^{\frac{1}{\mathrm{p}}},
$$

причем правая часть неуменьиаема на множестве всех разбиений $\Delta_{n+s}$, имеюциих одно и то же фиксированное множество $\omega_{1}$. В частности,

$$
\sup _{\Delta_{\mathrm{n}+\mathrm{s}}} \lim _{\mathrm{m}_{1}, \mathrm{~m}_{2} \rightarrow \infty} \Theta_{0, \mathrm{p}}\left[\left(\mathrm{m}_{1}, \mathrm{n}\right) ;\left(\mathrm{m}_{2}, \mathrm{n}+\mathrm{s}\right)\right]=2,
$$

если точная верхияя грань берется по всевозможсным разбиениям $\Delta_{\mathfrak{n}+\mathrm{s}}$, ккоторых не менее двух узлов содержится в каждом интервале $\delta_{j}^{n}, u$

$$
\sup _{\Delta_{2 n}} \lim \Theta_{0, \mathrm{p}}\left[\left(\mathrm{m}_{1}, \mathrm{n}\right) ;\left(\mathrm{m}_{2}, 2 \mathrm{n}\right)\right]=1,
$$

если точная верхняя грань берется по всевозможным разбиениям $\Delta_{2 \mathrm{n}}$, у которых по одному узлу содержится в каждом интервале $\delta_{\mathrm{j}}^{\mathrm{n}}$.

Доказательство.

$$
\int_{0}^{1}\left|e_{n, n+s}^{m_{1}, m_{2}}(f ; x)\right|^{p} d x=\left(\sum_{j \in \omega_{1}}+\sum_{j \in \omega_{2}} \int_{x_{j-1}^{n}}^{x_{j}^{n}} \int_{n, n+s}^{m_{1}, m_{2}}(f ; x) \mid d x \leq\right.
$$




$$
\begin{gathered}
\leq \sum_{j \in \omega_{1}}\left|f\left(x_{j}^{n}\right)-f\left(x_{j-1}^{n}\right)\right|^{p} \int_{x_{j-1}^{n}}^{x_{j}^{n}}\left|H_{0, m_{1}}\left(h_{j}^{n} ; x_{j}^{n}-x\right)-H_{0, m_{2}}\left(h_{j}^{n} ; x_{j}^{n}-x\right)\right|^{p} d x+2^{p} \sum_{j \in O_{2}} h_{j}^{n} \leq \\
\leq \sum_{j \in \omega_{1}} 2^{p} \cdot 2 \int_{0}^{h_{j}^{n} / 2} t^{p} d t+2^{p} \sum_{j \in \omega_{2}} h_{j}^{n}=\frac{1}{p+1} \sum_{j \in \omega_{1}}\left(h_{j}^{n}\right)^{p+l}+2^{p} \sum_{j \in \omega_{2}} h_{j}^{n} .
\end{gathered}
$$

С другой стороны, если каждый интервал $\delta_{j}^{n}$, для $\mathrm{j} \in \omega_{2}$, содержит узлы $\mathrm{x}_{\mathrm{j}-1}^{n}+\varepsilon, \mathrm{x}_{\mathrm{j}}^{\mathrm{n}}-\varepsilon$, то с учетом леммы $\mathrm{A}$, при $\varepsilon \rightarrow \infty$, получим

$$
\lim _{m_{1}, m_{2} \rightarrow \infty} \cdot \sup _{\|f\| \leq 1} \int_{x_{j-1}^{n}}^{x_{j}^{n}}\left|e_{n, n+s}^{m_{1}, m_{2}}(f ; x)\right|^{p} d x>2^{p}\left(h_{j}^{n}-2 \varepsilon\right)=2^{p} h_{j}^{n}+O(\varepsilon) .
$$

Тем самым первые два соотношения данной теоремы доказаны. Для доказательства третьего поступим аналогично. Пусть $x_{i}^{2 n} \in \delta_{j}^{n}$. Тогда $x_{j-1}^{n}=x_{2 j-2}^{2 n}$, $x_{i}^{2 n}=x_{2 j-1}^{2 n}, x_{j}^{n}=x_{2 j}^{2 n} n$

$$
\int_{x_{j-1}^{n}}^{x_{j}^{n}}\left|e_{n, 2 n}^{m_{1}, m_{2}}(f ; x)\right|^{p} d x=\left(\int_{x_{2 j-2}^{2 n}}^{x_{2 j-1}^{2 n}}+\int_{x_{2 j-1}}^{x_{2 j}^{2 n}}\right)\left|e_{n, 2 n}^{m_{1, n} m_{2}}(f ; x)\right|^{p} d x \leq \frac{1}{2} \int_{0}^{h_{2 j-1}^{2 n} / 2} d x+\frac{1}{2} \int_{0}^{h_{2 j}^{2 n} / 2} d x=h_{j}^{n} .
$$

Остается воспользоваться леммой А. Теорема 3 доказана.

Перейдем теперь к оценке величины $\mathrm{e}_{n, n+s}^{m_{1}, m_{2}}(\mathrm{f} ; \mathrm{x})$ на множестве функций $\mathrm{f} \in \mathrm{C}^{1} \mathrm{c}\|\mathrm{f}\|^{(1)} \leq 1$.

Если интервал $\delta_{j}^{n}$ не содержит узлов разбиения $\Delta_{n+s}$, то тогда, в силу теорем 1 и 2 из [1], имеем следующие соотношения:

$$
\frac{\sqrt{3} h_{j}^{n}}{9\left(2+h_{j}^{n}\right)} \leq \sup _{\|f\|^{(1)} \leq 1}\left\|e_{n, n+5}^{m, 0}(f ; x)\right\|_{C\left[\delta_{j}^{n}\right]} \leq \frac{h_{j}^{n}}{2+h_{j}^{n}}=\lim _{m \rightarrow \infty}\left\|e_{n, n+s}^{m, 0}(f ; x)\right\|_{C\left[\delta_{j}^{n}\right]},
$$

$\sup _{\|f\|^{(1)} \leq 1}\left\|e_{n, n+s}^{m_{1}, m_{2}}(f ; x)\right\|_{C\left[\delta_{j}^{n}\right]} \leq \frac{h_{j}^{n}}{2+h_{j}^{n}}$ и $\sup _{\|f\|^{(1)} \leq 1}\left\|e_{n, n+s}^{m_{1}, m+1}(f ; x)\right\|_{C\left[\delta_{j}^{n}\right]}=\frac{h_{j}^{n}}{2+h_{j}^{n}}\left\|\varphi_{m}\right\|_{C[0,1]}$, где $\varphi_{\mathrm{m}}$ - функция, определенная равенством (5).

Пусть теперь $\mathrm{j} \in \omega_{2}$, т. е. интервал $\delta_{j}^{n}$ содержит узлы разбиения $\Delta_{n+\mathrm{s}}$. Обозначим через. $\widetilde{\mathrm{x}}_{j}^{\mathrm{n}+\mathrm{s}}$ тот из них, который ближе других расположен к точке $\mathrm{x}_{\mathrm{j}-0,5}^{\mathrm{n}}=\frac{\mathrm{x}_{\mathrm{j}-1}^{\mathrm{n}}+\mathrm{x}_{\mathrm{j}}^{\mathrm{n}}}{2}$, и пусть, для определенности, $\mathrm{x}_{\mathrm{j}-0,5}^{\mathrm{n}} \leq \widetilde{\mathrm{x}}_{\mathrm{j}}^{\mathrm{n}+\mathrm{s}}$.

Рассмотрим сначала случай $\mathrm{m}_{1}=\mathrm{m}_{2}=0$. Обозначим через $1_{\mathrm{j}, \mathrm{n}+\mathrm{s}}$ - ломаную с изломом в точке $\widetilde{\mathrm{x}}_{\mathrm{j}}^{1+s}$ и принимающую в точках $\mathrm{x}_{j-1}^{n}, \widetilde{\mathrm{x}}_{\mathrm{j}}^{\mathrm{n}+\mathrm{s}}, \mathrm{x}_{\mathrm{j}}^{\mathrm{n}}$ значения, равные соответственно $0, \widetilde{y}_{j}^{n+s}=a\left(\widetilde{h}_{j}^{n}\right), y_{j}^{n}=a\left(\widetilde{h}_{j}^{n}\right)-a\left(h_{j}^{n}-\widetilde{h}_{j}^{n}\right)$, где 
$\widetilde{\mathrm{h}}_{\mathrm{j}}^{\mathrm{n}}=\widetilde{\mathrm{x}}_{\mathrm{j}}^{\mathrm{n}+\mathrm{s}}-\mathrm{x}_{\mathrm{j}-1}^{\mathrm{n}}$. А через $\mathrm{l}_{\mathrm{j}, \mathrm{n}}-$ линейную на промежутке $\bar{\delta}_{\mathrm{j}}^{\mathrm{n}}$ функцию, принимающую на его концах те же значения, что и функция $1_{j, n+s}$. Тогда, в силу леммы В, приходим к неравенству

$$
\max _{x \in \delta_{j}^{n}}\left|e_{n, n+s}^{0,0}(f ; x)\right| \leq\left|1_{j, n}\left(\widetilde{x}_{j}^{n+s}\right)-1_{j, n+s}\left(\widetilde{x}_{j}^{n+s}\right)\right|=a\left(\widetilde{h}_{j}^{n}\right)-\frac{\widetilde{h}_{j}^{n}}{h_{j}^{n}}\left(a\left(\widetilde{h}_{j}^{n}\right)-a\left(h_{j}^{n}-\widetilde{h}_{j}^{n}\right)\right) .
$$

Для дальнейшего нам понадобится следующее вспомогательное утверждение об одном свойстве функции а из леммы В.

Лемма 1. Для любых $h$ и $h_{1}, \frac{h}{2} \leq h_{1} \leq h$, справедливо неравенство

$$
a\left(h_{1}\right)-\frac{h_{1}}{h}\left(a\left(h_{1}\right)-a\left(h-h_{1}\right)\right) \leq a\left(\frac{h}{2}\right) .
$$

Доказательство. Левую часть этого неравенства после элементарных преобразований запишем как функцию $g$ переменного $h_{1}$ при фиксированном $h$ :

$$
g\left(h_{1}\right)=\frac{2 h_{1}\left(h-h_{1}\right)(4+h)}{h\left(2+h_{1}\right)\left(2+h-h_{1}\right)} .
$$

Эта функция убывает на промежутке $\left[\frac{h}{2}, h\right]$. Поэтому

$$
\max _{h_{1} \in\left[\frac{h}{2}, h\right]} g\left(h_{1}\right)=g\left(\frac{h}{2}\right) \frac{2 h}{4+h}=a\left(\frac{h}{2}\right) .
$$

Лемма 1 доказана.

На основании этой леммы заключаем, что

$$
\sup _{\|\mathrm{f}\|^{(1)} \leq 1}\left\|\mathrm{e}_{\mathrm{n}, \mathrm{n}+\mathrm{s}}^{0,0}(\mathrm{f} ; \mathrm{x})\right\|_{C\left[\bar{\delta}_{j}^{\mathrm{n}}\right]} \leq \frac{2 h_{j}^{\mathrm{n}}}{4+\mathrm{h}_{\mathrm{j}}^{\mathrm{n}}}
$$

Причем знак равенства достигается в случае, когда узел $\widetilde{\mathrm{x}}_{\mathrm{j}}^{\mathrm{n}+\mathrm{s}}=\mathrm{x}_{\mathrm{j}-0,5}^{\mathrm{n}}$.

Рассмотрим теперь случай $\mathrm{m}_{2}=0, \mathrm{~m}_{1}=\mathrm{m}>0$. С учетом свойств (3) и (4) функции $\mathrm{H}_{0, m}$, в предположении, что $\mathrm{x}_{\mathrm{j}-0,5}^{\mathrm{n}} \leq \widetilde{\mathrm{x}}_{\mathrm{j}}^{\mathrm{n}+\mathrm{s}}$, находим

$$
\begin{gathered}
\max _{x \in\left[x_{j-1,5,5}^{n}, x_{j}^{n}\right]}\left|e_{n, n+s}^{m, 0}(f ; x)\right| \leq\left|y_{j}^{n} H_{0, m}\left(h_{j}^{n} ; \widetilde{x}_{j}^{n+s}\right)-1_{j, n+s}\left(\widetilde{x}_{j}^{n+s}\right)\right| \leq \\
\leq\left|1_{j, n}\left(\widetilde{x}_{j}^{n+s}\right)-1_{j, n+s}\left(\widetilde{x}_{j}^{n+s}\right)\right| \leq \frac{2 h_{j}^{n}}{4+h_{j}^{n}} .
\end{gathered}
$$

А для $\mathrm{x} \in\left[\mathrm{x}_{\mathrm{j}-1}^{\mathrm{n}}, \mathrm{x}_{\mathrm{j}-0,5}^{\mathrm{n}}\right]$ имеем

$$
\max _{x \in\left[x_{j-1}^{n}, x_{j-1,5}^{n}\right]}\left|e_{n, n+s}^{m, 0}(f ; x)\right| \leq\left|1_{j, n+s}\left(x_{j-0,5}^{n}\right)\right| \leq \frac{2 h_{j}^{n}}{4+h_{j}^{n}} .
$$

Таким образом, 


$$
\sup _{\| f\left(\|^{(1)} \leq 1\right.}\left\|e_{n, n+s}^{m, 0}(f ; x)\right\|_{C\left[\bar{\delta}_{j}^{n}\right]} \leq \frac{2 h_{j}^{n}}{4+h_{j}^{n}},
$$

причем знак равенства достигается, когда узел $\widetilde{\mathrm{x}}_{\mathrm{j}}^{\mathrm{n}+s}=\mathrm{x}_{\mathrm{j}-0,5}^{\mathrm{n}}$.

Соотношение (8) остается справедливым и в случае $\mathrm{m}_{\mathrm{i}} \geq 0, \mathrm{i}=1,2$, при условии, что для $\mathrm{j}=\overline{1, n}$ в интервале $\delta_{j}^{n}$ узел $\widetilde{\mathrm{x}}_{\mathrm{j}}^{\mathrm{n}+\mathrm{s}}$ совпадает с точкой $\mathrm{x}_{\mathrm{j}-0,5}^{\mathrm{n}}$.

Наконец, в случае $\mathrm{m}_{\mathrm{i}} \geq 0, \mathrm{i}=1,2$, с учетом леммы $\mathrm{A}$, имеем

$$
\left|\mathrm{e}_{n, n+s}^{m_{1}, m_{2}}(f ; x)\right|<a\left(\widetilde{h}_{j}^{n}\right) \leq a\left(h_{j}^{n}\right)=\frac{2 h_{j}^{n}}{2+h_{j}^{n}} \leq \frac{2 \delta^{n}}{2+\delta^{n}} .
$$

Таким образом, доказана следующая теорема.

Теорема 4. Для любых $\mathrm{m}_{\mathrm{i}} \geq 0, \mathrm{i}=1,2$,

$$
\sup _{\Delta_{\mathrm{n}+\mathrm{s}}} \Theta_{1, \infty}\left[\left(m_{1}, n\right) ;\left(m_{2}, \mathrm{n}+\mathrm{s}\right)\right]=\frac{2 \delta^{\mathrm{n}}}{2+\delta^{\mathrm{n}}}
$$

$B$ cryuae же, когда $\mathrm{m}_{2}=0$,

$$
\sup _{\Delta_{\mathrm{n}+\mathrm{s}}} \Theta_{1, \infty}\left[\left(m_{1}, n\right) ;(0, n+s)\right]=\frac{2 \delta^{n}}{4+\delta^{n}},
$$

причем точная верхнял грань реализуется на разбиениях $\Delta_{n+s}$, у которых в каждом интервале $\delta_{\mathrm{j}}^{\mathrm{n}}$ содержится по узлу, совпадающему с точкой $\mathrm{x}_{\mathrm{j}-1}^{\mathrm{n}} . B$ случае разбиения $\Delta_{\mathrm{n}}$ с равноотстоящими узлами правые части в двух предыдуцих равенствах равны $\frac{1}{\mathrm{n}+0,5} \cup \frac{1}{2 \mathrm{n}+0,5}$ соответственно.

Теорема 5. Дия любых $\mathrm{m}_{\mathrm{i}} \geq 0, \mathrm{i}=1,2, u \Delta_{\mathrm{n}} \subset \Delta_{\mathrm{n}+\mathrm{s}}$ справедливо следуюиее соотиомение

$$
\sup _{\Delta_{n+s}} \Theta_{1,1}\left[\left(m_{1}, n\right) ;\left(m_{2}, n+s\right)\right]=2-8 \ln \prod_{j=1}^{n}\left(1+\frac{h_{j}^{n}}{4}\right)<\sum_{j=1}^{n}\left(h_{j}^{n}\right)^{2} .
$$

В частности, при $\mathrm{s}=\mathrm{n}$, и все $\delta_{j}^{\mathrm{n}}$ содержст по узлу разбиения $\Delta_{2 \mathrm{n}}$,

$$
\Theta_{1,1}\left[\left(m_{1}, n\right) ;\left(m_{2}, 2 n\right)\right]=\sum_{j=1}^{n} \frac{\left(h_{j}^{n}\right)^{2}}{4+h_{j}^{n}} .
$$

Eсли же $\mathrm{h}_{\mathrm{j}}^{\mathrm{n}}=\frac{1}{\mathrm{n}}, \mathrm{j}=\overline{1, \mathrm{n}}$, то правые части в (9) и (10) можно заменипь на $\frac{1}{4 \mathrm{n}}$ $u \frac{1}{4 \mathrm{n}+1}$ cоответственно.

Доказательство. Из тождества (3) имеем для любых $\mathrm{m}>0$ и $\mathrm{s} \geq 0$ 


$$
\int_{x_{j-1}^{n}}^{x_{j}^{n}} s_{0, m}\left(f, \Delta_{n+s} ; x\right) d x=\int_{x_{j-1}^{n}}^{x_{j}^{n}} s_{0,0}\left(f, \Delta_{n+s} ; x\right) d x .
$$

Поэтому, в случае, когда интервал $\delta_{j}^{n}$ содержит один узел $\widetilde{x}_{j}^{n+s}$ разбиения $\Delta_{\mathrm{n}+\mathrm{s}}, \mathrm{c}$ учетом леммы 1 и обозначений, введенных выше, находим

$$
\begin{gathered}
\int_{x_{j-1}^{n}}^{x_{j}^{n}}\left|e_{n, n+s}^{m_{1}, m_{2}}(f ; x)\right| d x=\int_{x_{j-1}^{n}}^{x_{j}^{n}}\left|e_{n, n+s}^{0,0}(f ; x)\right| d x=\left|1_{j, n}\left(\widetilde{x}_{j}^{n+s}\right)-1_{j, n+s}\left(\widetilde{x}_{j}^{n+s}\right)\right| \cdot \frac{h_{j}^{n}}{2} \leq \\
\leq a\left(\frac{h_{j}^{n}}{2}\right) \cdot \frac{h_{j}^{n}}{2}=\frac{h_{j}^{n}}{4+h_{j}^{n}} .
\end{gathered}
$$

Тем самым вторая часть этой теоремы доказана. Далее нам понадобится еще одно вспомогательное утверждение. Для $\frac{\mathrm{h}}{2} \leq \beta \leq \mathrm{h}$ положим

$$
\omega(h, \beta ; x)= \begin{cases}\frac{2 x}{2+x}, & 0 \leq x \leq \beta, \\ \frac{2(h-x)}{2+h-x}+4 \frac{2 \beta-h}{(2+\beta)(2+h-\beta)}, & \beta \leq x \leq h,\end{cases}
$$

и

$$
l(h, \beta ; x)=\frac{4 x}{h} \cdot \frac{2 \beta-h}{(2+\beta)(2+h-\beta)}
$$

Лемма 2. $\int_{0}^{\mathrm{h}}(\omega(\mathrm{h}, \beta ; \mathrm{x})-1(\mathrm{~h}, \beta ; \mathrm{x})) \mathrm{dx} \leq \int_{0}^{\mathrm{h}} \omega\left(\mathrm{h}, \frac{\mathrm{h}}{2} ; \mathrm{x}\right) \mathrm{dx}=2 \mathrm{~h}-8 \ln \left(1+\frac{\mathrm{h}}{4}\right)<\mathrm{h}^{2}$.

Доказательство. Легко вычислить

$$
\begin{gathered}
\int_{0}^{h}(\omega(h, \beta ; x)-1(h, \beta ; x)) d x= \\
=2 h+8 \ln 2-2\left(2 \ln (2+\beta)(2+h-\beta)+\frac{(2 \beta-h)^{2}}{(2+\beta)(2+h-\beta)}\right) .
\end{gathered}
$$

Правая часть этого равенства при фиксированном һ представляет собой функцию переменной $\beta$. Непосредственной проверкой убеждаемся, что эта функция убывает на промежутке $\left[\frac{\mathrm{h}}{2}, \mathrm{~h}\right]$, что и доказывает лемму 2.

Заметим, что $\omega(\mathrm{h}, \beta ; \mathrm{h})=4 \cdot \frac{2 \beta-\mathrm{h}}{(2+\beta)(2+h-\beta)}$ 
при фиксированном $h$ представляет собой непрерывную функцию переменной $\beta$, которая на промежутке $\left[\frac{\mathrm{h}}{2}, \mathrm{~h}\right]$ строго возрастает от значения $\omega\left(\mathrm{h}, \frac{\mathrm{h}}{2} ; \mathrm{h}\right)=0$ до значения $\omega(\mathrm{h}, \mathrm{h} ; \mathrm{h})=\frac{2 \mathrm{~h}}{2+\mathrm{h}}$. Поэтому любому значению $\mathrm{y} \in\left[0, \frac{2 \mathrm{~h}}{2+\mathrm{h}}\right]$ отвечает единственное значение $\beta=\beta(\mathrm{y})$ такое, что $\omega(\mathrm{h}, \beta(\mathrm{y}) ; \mathrm{h})=\mathrm{y}$.

Перейдем теперь к доказательству первой части теоремы (5). Пусть $f \in \mathrm{C}^{l}$ и $\|\mathrm{f}\|^{(1)} \leq 1$. Для фиксированных $\mathrm{j}, \mathrm{j}=\overline{1, n}$, при рассмотрении функции $\mathrm{e}_{n, n+s}^{m_{1}, m_{2}}(f ; x)$ можно, не уменьшая обццности, считать, что $f\left(x_{j-1}^{n}\right)=0$, $\mathrm{f}\left(\mathrm{x}_{\mathrm{j}}^{\mathrm{n}}\right)=\mathrm{y}_{\mathrm{j}}^{\mathrm{n}} \geq 0$. Воспользуемся равенством (11) и утверждениями леммы В и леммы 2:

$$
\begin{gathered}
\int_{x_{j-1}^{n}}^{x_{j}^{n}}\left|e_{n, n+s}^{m_{1}, m_{2}}(f ; x)\right| d x=\int_{x_{j-1}^{n}}^{x_{j}^{n}}\left|\omega\left(h_{j}^{n}, \beta\left(y_{j}^{n}\right) ; x\right)-1\left(h_{j}^{n}, \beta\left(y_{j}^{n}\right) ; x\right)\right| d x \leq \\
\leq \int_{x_{j-1}^{n}}^{x_{j}^{n}} \omega\left(h_{j}^{n}, \frac{h_{j}^{n}}{2} ; x\right) d x=2 h_{j}^{n}-8 \ln \left(1+\frac{h_{j}^{n}}{4}\right)<\left(h_{j}^{n}\right)^{2} .
\end{gathered}
$$

Откуда получаем оценку сверху

$\int_{0}^{1}\left|e_{n, n+s}^{m_{1}, m_{2}}(f ; x)\right| d x \leq \sum_{j=1}^{n}\left(2 h_{j}^{n}-8 \ln \left(1+\frac{h_{j}^{n}}{4}\right)\right)=2-8 \ln \prod_{j=1}^{n}\left(1+\frac{h_{j}^{n}}{4}\right)<$ $<\sum_{j=1}^{n}\left(h_{j}^{n}\right)^{2}$

С другой стороны, при $\delta^{\mathrm{n}+\mathrm{s}} \rightarrow 0, \mathrm{~s} \rightarrow \infty$, $\sup \left\{\int_{0}^{1}\left|e_{n, n+s}^{m_{1}, m_{2}}(f ; x)\right| d x: f \in C^{1},\|f\|^{(1)} \leq 1, f\left(x_{j}^{n}\right)=0\right\} \rightarrow \sum_{j=1}^{n} \int_{x_{j-1}^{n}}^{x_{j}^{n}} \omega\left(h_{j}^{n}, \frac{h_{j}^{n}}{2} ; x\right) d x$.

Таким образом, теорема 5 доказана.

\section{Библиографнческие ссылки}

1.Великин В.Л. Точные значения и оценки интерполяционных растворов некоторых подпространств эрмитовых сплайнов / В.Л. Великин // Вісник Дніпропетр. ун-ту. Математика. - 2009, - Т.17, №6/1, вип. 14, С.54-56.

2.Великин В.Л. Точные значения приближения эрмитовыми сплайнами на классе дифференцируемых функций / В.Л. Великин // Изв. АН СССР. Серия математическая, $-1973 .-37,-$ C.165-185. 